Now the idea of a vector is one entirely foreign to ordinary algebra, in which the square of any unit is positive unity. Hence the fact that the square of a unit vector is negative unity has no disadvantage. It makes the scalar part of the product of $\beta$ into $\alpha$ equal to the product of the lengths of these vectors into the cosine of the supplement of the angle between their positive directions; and it makes the reciprocal of a vector have a direction opposite to that of the vector itself; all of which conditions are as natural and simple as their opposites.

Finally, it is shown by Hamilton, by strict reasoning (Lectures, $\$ \S 49-56$ ), that these laws for the multiplication of unit rectangular vectors must hold if no one direction in space is to be regarded as eminent above another and if the ordinary rules of algebra are to apply in so far that, (1) to multiply either factor by any number positive or negative, multiplies the product by the same, (2) the product of two determined factors is itself determined, (3) the distributive and associative principles hold. We see then that Hamilton's system is one which preeminently satisfies the conditions of correspondence to ordinary algebra as far as possible.

\title{
Note on a Problem in Analytical Geometry.
}

By A. J. Pressland, M.A.

\section{[Abstract. $]$}

The theorem, "If upon the sides of a triangle as diagonals parallelograms be described, whose sides are parallel to two given lines, then the other three diagonals will intersect in the same point," occurs in Hutton's Course of Mathematics, 12th ed., vol. II., p. 191.

For a proof, see Smith's Conic Sections, p. 40.

If we are given the point of intersection of the diagonals, and wish to find the directions of the sides of the parallelograms, the discussion resolves itself into describing a conic through three points to have its centre at a given point. The asymptotes of this conic are the directions required. For a solution, see Eagles' Constructive Geometry of Plane Curves, pp. 124, 173, and notice Taylor, Ancient and Modern Geometry of Conics, p. 164, Ex. 454.

If $\mathrm{A}, \mathrm{B}, \mathrm{C}$ be the three points, $\mathrm{D}, \mathrm{E}, \mathrm{F}$ the mid points of $\mathrm{BC}, \mathrm{CA}$, $\mathrm{AB}$ then if the centre lies inside DEF the asymptotes are imaginary, but they are real if the centre lies inside AEF, etc. 
From the theorem, the following proof of the nine-point circle is obtainable (Fig. 27).

Take any line CK, and draw rectangles as in the figure, we have,

$$
\angle \mathrm{MPK}=\angle \mathrm{PMC}-\angle \mathrm{DKC}=\angle \mathrm{ECM}-\angle \mathrm{DCK}=\angle \mathrm{EFD},
$$

therefore $\mathrm{F}, \mathrm{P}, \mathrm{D}, \mathrm{E}$ are concyclic. If $\mathrm{KD}$ is perpendicular to $\mathrm{AC}$, $P M$ is perpendicular to $\mathrm{BC}$, and their intersection is the mid point of $\mathrm{CO}$, where $\mathrm{O}$ is the orthocentre. If $\mathrm{CK}$ and $\mathrm{CB}$ coincide, $\mathrm{P}$ is the foot of the perpendicular from $\mathrm{A}$ on $\mathrm{BC}$.

Seventh Meeting, May 12, 1893.

Joun Alison, Esq, M.A., F.R.S.E., President, in the Chair.

\section{On the History of the Fourier Series.}

By George A. Gibson, M.A.

$\S 1$. The treatment of the Fourier Series, that is, of the series which proceeds according to sines and cosines of multiples of the variable, is in most English text-books very unsatisfactory; in many cases it shows almost no advance upon that of Poisson and, even where a more or less accurate reproduction of Dirichlet's investigations is given, there is no attempt at indicating the advantages it possesses over the so-called proof of Poisson. Nor is the uniformity of the convergence of the series so much as mentioned, not to siay discussed. I have therefore thought it might be useful to give a fairly complete outline of the historical development of the series so far as the materials at my disposal allow. I do not think that any important contribution to the theory is omitted, but, as I indicate at one or two places, there are some memoirs to which I have not had access and which I only know at second hand.

Again it is to be understood that only series of the form

$$
A_{0}+\sum_{n-1}^{n-\infty}\left(A_{n} \cos n x+B_{n} \sin n x\right)
$$

$n$ being an integer, are dealt with, those cases in which $n$ is not integral being omitted in the meantime. 